\title{
Non-equilibrium Goldstone phenomenon in tachyonic preheating
}

\author{
Sz. Borsányi*, A. Patkós †and D. Sexty ${ }^{\ddagger}$ \\ Department of Atomic Physics \\ Eötvös University, Budapest, Hungary
}

June 11, 2018

\begin{abstract}
The dominance of the direct production of elementary Goldstone waves is demonstrated in tachyonic preheating by numerically determining the evolution of the dispersion relation, the equation of state and the kinetic power spectra for the angular degree of freedom of the complex matter field. The importance of the domain structure in the order parameter distribution for the quantitative understanding of the excitation mechanism is emphasized. Evidence is presented for the very early decoupling of the low-momentum Goldstone modes.
\end{abstract}

\section{Introduction}

The aim of this investigation is to contribute to the systematic exploration of the transition from the inflationary evolution of the Universe to the standard cosmological regime. The numerical analysis was performed in a simple hybrid inflationary model, in which the inflaton is coupled to a complex scalar field [1, 2]. The equations of the fields and of the scale parameter of Friedmann-RobertsonWalker (FRW) geometry were solved simultanously. The characteriztics of the transition were studied for a range of couplings and initial conditions. All choices satisfy the cosmological constraints entailing a sufficient number of e-foldings during inflation and the generation of density perturbations compatible with the measured cosmic microwave background radiation (CMBR) anisotropy.

The present investigation is focused on the excitation of Goldstone modes. In the literature the decay of global cosmic strings is advocated as the main source of these particles [3, 4, 5, 6. It will be demonstrated that in the period of tachyonic instability [7, 8] a dominant direct Goldstone generation takes place. The importance of Goldstone production was first emphasized by Boyanovsky et al. 9, 10, in a renormalized large- $N$ approach to the quantum dynamics of the symmetry breaking in the $\mathrm{O}(\mathrm{N})$ model. They have extended their investigation to the FRW geometry in the framework of the new inflationary scenario [1]. The present investigation is an extension of our study of the

\footnotetext{
*mazsx@cleopatra.elte.hu

$\dagger$ patkos@ludens.elte.hu

‡denes@achilles.elte.hu
} 
classical $O(N)$ system in Minkowski metrics published in Ref. 12 to the case of FRW-geometry. In the present paper also the period of instability is treated classically. Although the time interval of the instability is rather short it is instructive to study the transition of the scale parameter of the Universe from the inflationary regime to a regime dominated by the mixture of weakly interacting species. We study the field dynamics under continous variation of the power characterizing the time dependence of the cosmological scale factor.

In hybrid inflationary scenarios the rolling inflaton triggers the condensation of a complex scalar field thought to be the matter field driving the grand unified theory (GUT) phase transition. The transition is accompanied by spinodal (tachyonic) instabilities, which occur for the radial (in the present case, $O(2)$ invariant) modes. Rising radial (Higgs) modes excite on their turn massless angular (Goldstone) modes. We refer to this phenomenon as the nonequilibrium Goldstone effect. It was tested by systematically varying the lattice spacing that the excitation process is rather insensitive to the details of the discretization.

After the tachyonic instability is stopped one observes an excess in the gradient energy density of the Goldstone degree of freedom relative to the corresponding kinetic energy density which is argued in [5] to be the signature for the occurrence of finite density of global strings. Evidence will be presented for the importance of topological configurations (domain walls and/or strings) in causing this difference, which is sustained over a considerable time interval. Also, a clean radiative equation of state $(\mathrm{EoS})$ is found for the Goldstone degree of freedom after the virial equilibrium is reached. After further evolution, however, a clear two-component separation was observed in the Goldstone power spectra. Low-(comoving)- $k$ modes decouple from the equilibration processes fairly early and expand further as a noninteracting massless radiation with a frozen momentum distribution. High- $k$ modes interact with the massive (Higgs and inflaton) modes. In this range we observe quantitative lattice spacing dependence, which does not challenge the qualitative features of the emerging physical picture. The size of the decoupled comoving $k$ interval of the Goldstone excitations increases with time.

In Section 2 the model is presented and the setting of the initial conditions is discussed. Their choice is crucial to ensure the minimal sensitivity of the results to the spatial discretization. The most important features of the excitation process of Goldstone modes, shortly outlined in the previous paragraph, are discussed in Section 3. A simple estimate will also be given there for the ratio of the direct Goldstone production relative to the energy contained in extended objects. The features and the limitations of a semianalytical model for the excitation of the different modes is discussed in Section 4. Section 5 is devoted to the discussion of the late-time expansion. Conclusions are summarized in Section 6.

\section{Selection of couplings and initial conditions}

The system consists of the real (harmonic) inflaton field $\sigma(\mathbf{x}, t)$ and the complex matter field $\phi(\mathbf{x}, t)$, with negative squared mass and a quartic stabilizing $O(2)$ invariant self-interaction. Their evolution is treated self-consistently assuming a spatially flat FRW space-time characterized by its dynamically determined scale function $a(t)$. Metric fluctuations are beyond the scope of the present study. The equations describing the field dynamics of the system are

$$
0=\ddot{\sigma}(\mathbf{x}, t)+3 H \dot{\sigma}(\mathbf{x}, t)-\Delta \sigma(\mathbf{x}, t)+m_{\sigma}^{2} \sigma(\mathbf{x}, t)+g^{2}|\phi(\mathbf{x}, t)|^{2} \sigma(\mathbf{x}, t),
$$




$$
0=\ddot{\phi}(\mathbf{x}, t)+3 H \dot{\phi}(\mathbf{x}, t)-\Delta \phi(\mathbf{x}, t)+m^{2} \phi+\frac{\lambda}{6}|\phi(\mathbf{x}, t)|^{2} \phi(\mathbf{x}, t)+g^{2} \sigma^{2}(\mathbf{x}, t) \phi(\mathbf{x}, t) .
$$

The FRW equation has the following form:

$$
H^{2}-\frac{8 \pi}{3 m_{p l}^{2}}\left[\rho_{\text {Higgs }}(\mathbf{x}, t)+\rho_{\text {Goldstone }}(\mathbf{x}, t)+\rho_{\text {inflaton }}(\mathbf{x}, t)\right]=0 .
$$

In Eqs. (11) and (2) the Hubble parameter $H=\dot{a}(t) / a(t)$ was introduced and $m_{p l}$ is the Planck mass. In the second term of the left hand side of Eq. (2) the expression in the square brackets represents the microscopical energy density of the physical decomposition of the fields, to be identified below. The explicit expressions will appear in Eqs. (8)-(10).

For the numerical simulation the field equations were rewritten in conformal time. For instance, in the $O(2)$ sector one has

$$
\psi^{\prime \prime}-\Delta \psi-\frac{a^{\prime \prime}}{a} \psi+m^{2} a^{2} \psi+\frac{\lambda}{6}|\psi|^{2} \psi+g^{2} \Sigma^{2} \psi=0,
$$

with $\psi=a(t) \phi, \Sigma=a(t) \sigma$, and both time and space coordinates are measured in proportion to the scale factor $\left[\left(d \eta=d t / a(t), d x=d x_{p h y s} / a(t)\right)\right]$. Finally, we scale all quantities once more with an appropriate power of the dimensionless combination $m_{d} a_{i n i t}$, where $m_{d}$ is an arbitrary mass unit and $a_{\text {init }}$ equals to the scale factor at the beginning of the simulation $\left[a_{\text {init }}=a\left(t_{\text {init }}\right)\right.$, see below]:

$$
\psi_{\text {lat }}^{\prime \prime}-\Delta \psi_{\text {lat }}-\frac{a^{\prime \prime}}{a} \psi_{\text {lat }}+\frac{m^{2} a^{2}}{m_{d}^{2} a_{\text {init }}^{2}} \psi_{\text {lat }}+\frac{\lambda}{6}\left|\psi_{\text {lat }}\right|^{2} \psi_{\text {lat }}+g^{2} \Sigma_{\text {lat }}^{2} \psi_{\text {lat }}=0,
$$

The physical quantities are expressed through those simulated on the lattice as follows:

$$
\phi=\frac{\psi}{a}=\psi_{\text {lat }} m_{d} \frac{a_{\text {init }}}{a} \quad d x_{\text {phys }}=d x a=\frac{a}{a_{\text {init }}} \frac{d x_{\text {lat }}}{m_{d}} \quad d t=a d \eta=\frac{a}{a_{\text {init }}} \frac{d \eta_{\text {lat }}}{m_{d}}
$$

A convenient choice is to set $m_{d}=|m|$. In the following, we always use the powers of $|m|$ as units of measurement. As one can check, also in the scaled FRW equation only $a / a_{\text {init }}$ appears, plus the ratio of the Planck mass and the $O(2)$ mass parameter. Therefore one can study the evolution of $a(t) / a_{\text {init }}$, independently of the choice of $m_{d}$.

After this scaling, we discretized the equations of the quantities labeled by "lat" in a comoving volume $(L / a(t))^{3}$, with $L=N \delta x_{\text {lat }} /|m| \times a(t) / a_{\text {init }}, N=64,128, \delta x_{\text {lat }}=1$. Here $t_{\text {init }}$ is the time instant where we start the numerical solution of Eqs. (11) and (2), which has been conveniently choosen to slightly preceed the exit point from the inflation. In the plots to be presented below the time is measured relative to $t_{\text {init }}$. The insensitivity of the results to the lattice spacing was tested by also employing $\delta x_{l a t}=0.25,0.5,0.75$. The conformal time step $\delta \eta$ was chosen in proportion to the spatial lattice spacing $\delta x$ in the range $1 / 16-1 / 64$.

On the other hand, we find for the physical extent of our system $N \delta x_{\text {lat }} / m_{d} \times a(t) / a_{\text {init }} \ll H^{-1}(t)$. Therefore we actually study only a small portion of the volume of the whole Universe. This is different than the choice of the lattice spacing in Ref. [13, where the system is at least as large as one Hubble volume. Therefore we do not expect the string part of the Goldstone dynamics to be described truly faithfully, but the propagating quasiparticle excitations are well represented in the simulations.

We will be able to argue convincingly that in the investigation of the early appearance of Goldstone modes, the effect of the spatial cutoff will not cause any finite lattice spacing distortion. The choice of 
the lattice constant, however, is significant for the decay of the Higgs waves into Goldstone excitations. This process is energetically allowed as long as its comoving mass $m_{H} a(t) / a_{\text {init }}$ is smaller than twice the maximal allowed comoving momentum for the Goldstone waves on the lattice $k_{\text {cutoff }}$. With increasing redshift one arrives at an artificial stabilization of the Higgs waves. Therefore, at best, qualitative features of the late time evolution of the system are expected to be physical.

The matter field started in the symmetric phase, in the close vicinity of the point $\phi_{0}\left(t_{\text {init }}\right) \equiv$ $V^{-1} \int d^{3} x \phi\left(\mathbf{x}, t_{\text {init }}\right)=0$. The initial Higgs velocity is $\dot{\phi}_{0}\left(t_{\text {init }}\right)=0$. For the homogenous inflaton mode the amplitude and velocity values in the moment $t=t_{\text {init }}$ were drawn from the solution of the equations describing its roll down, started at the Planck scale: $\sigma_{0} \equiv V^{-1} \int d^{3} x \sigma(\mathbf{x}, t=0)=m_{p l}$.

Two important cosmological constraints are to be satisfied. The first requires at least $N_{T O T} \sim$ 60 e-foldings of the scale factor before the critical inflaton field value is reached which terminates inflation. The other constraint stems from the relation of the quantum fluctuations of the inflationary period to the density fluctuations measured by the COBE experiment 14. The numerical method of selecting the couplings without relying on the slow-roll approximation was described in some detail in Ref. [17.

In the present investigation the GUT scale was chosen for the scale of the end of inflation. Therefore for the quantity $m_{H}^{2} \equiv-2 m^{2}$, variation in the region $m_{H} \sim 10^{(14-15)} \mathrm{GeV}$ was allowed. The inflaton-Higgs coupling was varied in the interval $g=0.01-0.1$. The value of the Higgs selfcoupling was fixed with the relation $\lambda=3 g^{2}$, which is valid if the couplings of the hybrid theory are derived from a superpotential [15, 16]. The detailed numerical analysis was performed with $g=$ $0.1, m_{H}=8.8 \times 10^{14} \mathrm{GeV}, m_{\sigma}=4.2 \times 10^{11} \mathrm{GeV}, N_{T O T}=60$, and $g=0.01, m_{H}=5.5 \times 10^{14} \mathrm{GeV}, m_{\sigma}=$ $1.4 \times 10^{12} \mathrm{GeV}, N_{T O T}=60$.

The initial population of the inhomogenous modes imitates the quantum vacuum. The variation of the lattice spacing changes the size of the Brillouin-zone, therefore under such variation one would simulate systems with different cosmological constants. Using for the Fourier mode functions the complete orthonormal set $1 /\left(N \delta x_{l a t}\right)^{3 / 2} e^{i \mathbf{k x}}$, one finds nearly lattice spacing independent energy densities if modes with $|k| \leq k_{\max }$ are filled as follows:

$$
\begin{gathered}
\sigma_{k}\left(t_{e}\right)=\sqrt{\frac{1}{2 \omega_{\sigma}}} e^{i \alpha_{k}}, \quad \dot{\sigma}_{k}\left(t_{e}\right)=-i \sqrt{\frac{\omega_{\sigma}}{2}} e^{i \beta_{k}}, \quad \omega_{\sigma}^{2}=k^{2}+m_{\sigma}^{2}, \\
\phi_{k}\left(t_{e}\right)=\sqrt{\frac{1}{2 \omega_{\phi}}} e^{i \gamma_{k}}, \quad \dot{\phi}_{k}\left(t_{e}\right)=-i \sqrt{\frac{\omega_{\phi}}{2}} e^{i \delta_{k}}, \quad \omega_{\phi}^{2}=k^{2}+m^{2}+g^{2} \sigma^{2}\left(k=0, t_{e}\right) .
\end{gathered}
$$

The initial phases $\alpha_{k}, \beta_{k}, \gamma_{k}, \delta_{k}$ were chosen randomly. It was tested that our conclusions are not sensitive to the choice of the maximal filled momentum states, e.g $k_{\max } \in(2 ., 3$.). In order to have very accurate equality of the initial energy densities in case of different lattice spacings, the inhomogenous modes were filled only up to a lattice spacing dependent maximal comoving wave number $k_{\text {max }}\left(\delta x_{\text {lat }}\right)$. For instance, when choosing $k_{\max }=2$ for $\delta x_{l a t}=0.5, N=128$, accurate matching of the initial energy densities was achieved for $k_{\max }=1.84$ on the lattice $\delta x_{\text {lat }}=1.0, N=64$. The difference in $k_{\max }$ comes from the distortion of the energy-momentum relation on lattice. (Be conscious also of the other restriction, that during inflation the energy content of the inhomogeneous modes should be much smaller than the potential energy of the $O(2)$ field, e.g. false vacuum domination).

With this choice we have normalised the energy density to the same value for any lattice spacing, which leads to finite final average energy densities when $\delta x_{l a t}$ is diminished [18. On the other hand, no further renormalization was necessary to reach lattice spacing independent conclusions concerning the excitation process. 


\section{Direct Goldstone excitation via spinodal instability}

The independent degrees of freedom. The time evolution of the normalized cross-correlation matrix introduced in [12] presents evidence that directly after the spinodal instability is over, one can choose for the three independent degrees of freedom the inflaton, the radial $\mathrm{O}(2)$ invariant motion of $r(\mathbf{x}, t)=|\phi(\mathbf{x}, t)|$ and the angular oscillations $\varphi(\mathbf{x}, t),\left(\phi=r e^{i \varphi}\right)$. The time evolution of the dispersion relation characterizing these degrees of freedom was calculated from the definition

$$
\omega_{k}^{2}=\frac{\left|\dot{X}_{k}\right|^{2}}{\left|X_{k}\right|^{2}}, \quad X_{k}=\sigma_{k}, r_{k},\left(e^{i \varphi}\right)_{k},
$$

and extrapolated to $k=0$ for finding the corresponding masses [12, 17. One obtains for the angular phase factor shortly after the spinodal instability mass values which are compatible with zero within the error of the mass determination. This observation justifies the term "Goldstone" for the angular modes. In the following the radial degree of freedom will be simply referred to as Higgs, and the angular component as Goldstone.

The phase transition triggered by the inflaton field can be clearly seen on the time evolution of the homogeneous mode $\bar{r}^{V}(t)$ of the Higgs field (the overline with index "V" means spatial averaging). The tachyonic instability leads to an almost instantanous exponential switch into the symmetry broken regime as shown in Fig. 1. The Higgs field triggers the rise of the gradient energy of the Goldstone component with a slight delay and the increase of the Goldstone kinetic energy starts with a further delay. The sharp increase in the radial component is terminated by an oscillatory period, whose frequency is determined by the sum of the classical mass square around the minimum $r_{0}$ of the potential and the space average of the Higgs fluctuations: $-2 m^{2}+\lambda \bar{r}^{V} / 2$, which is often referred to as the Hartree mass. It is clear from the figure that the oscillations in the Goldstone kinetic and gradient energies forcefully follow the same frequency.

In Fig. 2 we analyze the time dependence of the average energy densities. The twin curves, which refer to solutions in the same physical volume, but with different lattice spacings illustrate to what extent the dynamics of different degrees of freedom depends on the details of discretization during spinodal instability. The Higgs component starts to vary first, reflecting the instability. The inflaton and the Goldstone energy densities follow it with approximately equal delay. Clearly, the excitation of these modes is driven by the temporal variation of the Higgs field $r(\mathbf{x}, t)$ (see section 4). Fig. 2 also suggests that for $g=0.1$ the three motions are apparently decoupled from each other after the out-of-equilibrium oscillations are damped, each having its own nearly constant density.

The Goldstone field reaches the highest energy density $\sim 30|m|^{4}$, which is slightly higher than the energy density corresponding to the one-loop estimate of the critical temperature for an $O\left(N_{\text {comp }}=2\right)$ model, that is $\sqrt{72 /\left(N_{\text {comp }}+2\right) \lambda}|m| \sim 25|m|$, with $\lambda=0.03$. The other two degrees of freedom are much colder. Instant "freezing" characterizes the behavior of the inflaton field. It obtains a rather large squared mass, nearly equal to the Higgs mass, due to the supersymmetric $\lambda-g^{2}$ relation (e.g. $\left.m_{\sigma}^{2}-6 g^{2} m^{2} / \lambda \approx-2 m^{2}=m_{H}^{2}\right)$. One calculates the potential energy of the inflaton with its "new" mass when checking the virial equilibrium in this degree of freedom. The sudden increase of its full energy density can be semi-quantitatively understood to be the result of this mass change (see section 4).

On the right edge of Fig. 2 the effect of the late-time expansion appears: the energy density of the inflaton field is hardly varying which corresponds to its nonrelativistic nature. On the other hand the energy density ratio of the Higgs and the Goldstone fields stays nearly constant for $t \leq 5000$. 


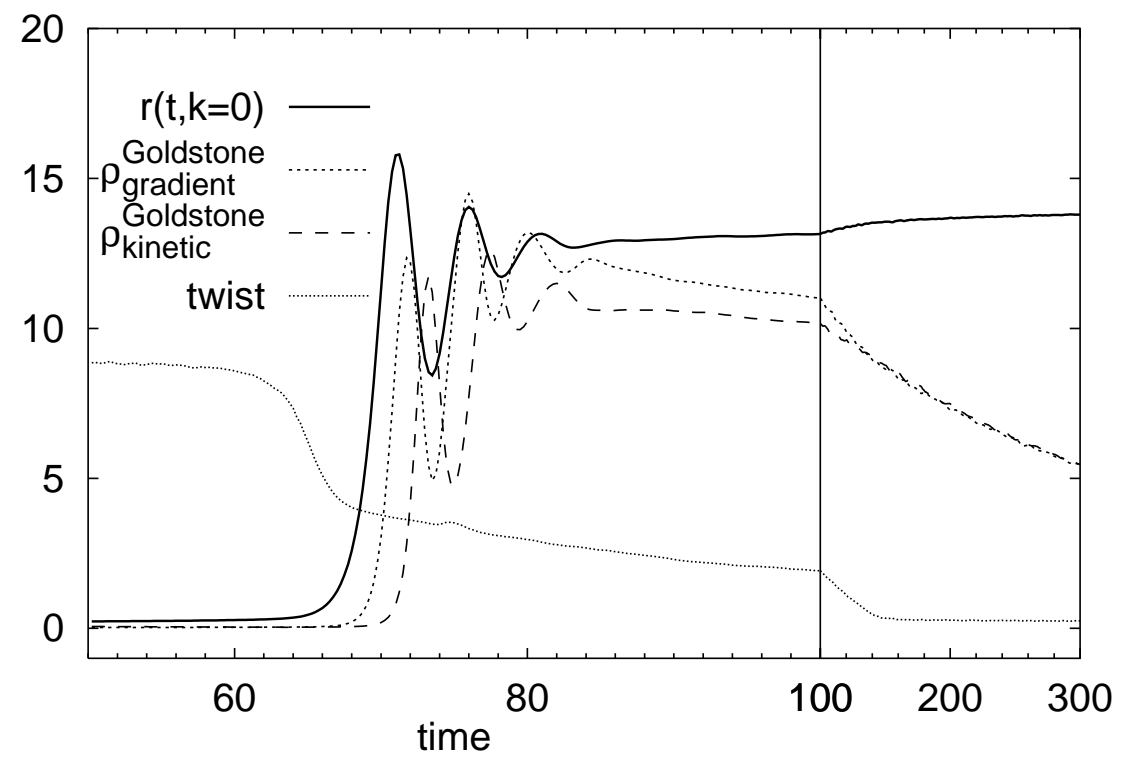

Figure 1: Virialization of the Goldstone oscillations, and the evolution of the average of the integrated twist in $\varphi(\mathbf{x}, t)$, calculated along straight lattice lines parallel to one of the axes. The oscillations of the radial field $\overline{r(\mathbf{x}, t)}^{V}$ drive the variation of the gradient and kinetic energy densities of the Goldstone modes $\left(g=0.1, \lambda=3 g^{2}, N=64\right)$.

We shall return to the discussion of the apparently coupled cooling of the radial and of the angular $O(2)$ components in section 5 .

The equations of state (EoS). We observed that the system stays in a rather stable way deep in the broken symmetry phase, despite the fact that the Goldstone "temperature" is high. One might attempt in such circumstances to characterize each species as a noninteracting (decoupled) gas, possessing its own equation of state. The local energy densities and pressures have the following expressions:

$$
\begin{gathered}
\rho_{\text {Higgs }}(\mathbf{x}, t)=\frac{1}{2} \dot{r}(\mathbf{x}, t)^{2}+\frac{1}{2}(\nabla r(\mathbf{x}, t))^{2}+\frac{3}{2} \frac{m^{4}}{\lambda}+\frac{1}{2} m^{2} r(\mathbf{x}, t)^{2}+\frac{\lambda}{24} r(\mathbf{x}, t)^{4}, \\
p_{\text {Higgs }}(\mathbf{x}, t)=\frac{1}{2} \dot{r}(\mathbf{x}, t)^{2}-\frac{1}{6}(\nabla r(\mathbf{x}, t))^{2}-\frac{3}{2} \frac{m^{4}}{\lambda}-\frac{1}{2} m^{2} r(\mathbf{x}, t)^{2}-\frac{\lambda}{24} r(\mathbf{x}, t)^{4}, \\
\rho_{\text {Goldstone }}(\mathbf{x}, t)=\frac{1}{2} r(\mathbf{x}, t)^{2} \dot{\varphi}(\mathbf{x}, t)^{2}+\frac{1}{2}(\nabla \varphi(\mathbf{x}, t))^{2}, \\
p_{\text {Goldstone }}(\mathbf{x}, t)=\frac{1}{2} r(\mathbf{x}, t)^{2} \dot{\varphi}(\mathbf{x}, t)^{2}-\frac{1}{6} r(\mathbf{x}, t)^{2}(\nabla \varphi(\mathbf{x}, t))^{2}, \\
\rho_{\text {inflaton }}(\mathbf{x}, t)=\frac{1}{2} \dot{\sigma}(\mathbf{x}, t)^{2}+\frac{1}{2}(\nabla \sigma(\mathbf{x}, t))^{2}+\frac{1}{2} g^{2} \sigma(\mathbf{x} \cdot t)^{2} r(\mathbf{x}, t)^{2}, \\
p_{\text {inflaton }}(\mathbf{x}, t)=\frac{1}{2} \dot{\sigma}(\mathbf{x}, t)^{2}-\frac{1}{6}(\nabla \sigma(\mathbf{x}, t))^{2}-\frac{1}{2} g^{2} \sigma(\mathbf{x}, t)^{2} r(\mathbf{x}, t)^{2} .
\end{gathered}
$$




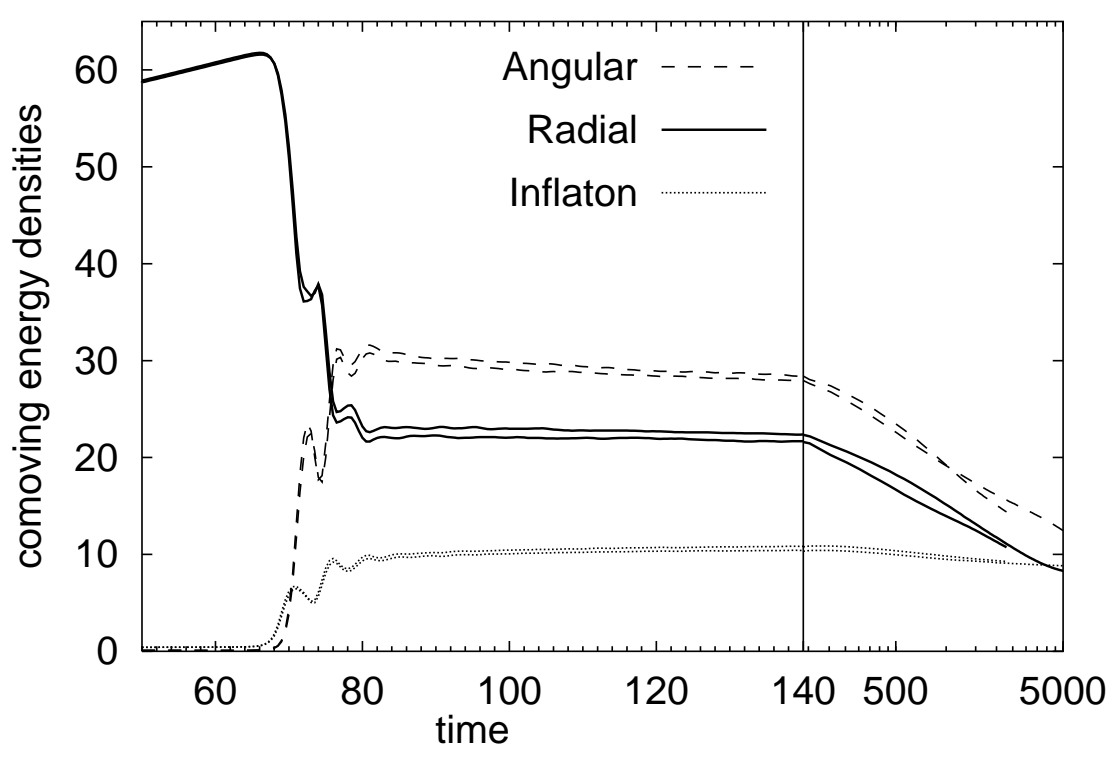

Figure 2: Average comoving energy densities $\left(\left(a^{2}(t) / a_{\text {init }}^{3}\right) \rho_{i}(t), i=G, H\right.$, inflaton $)$ of the independent degrees of freedom $\left(g=0.1, \lambda=3 g^{2}, N=64,\left(\delta x_{\text {lat }}=1\right), N=128,\left(\delta x_{\text {lat }}=0.5\right)\right)$. The figure represents the average of 8 runs for $N=64$ and 4 for $N=128$, each starting with random initial phases. The evolution for $t>140$ is plotted on a logarithmic time scale. The shorter curves correspond to the solutions on finer spatial lattice.

In the equations of state the space averages of the above expressions appear. Note that the Higgs inflaton interaction term is associated, with some arbitrariness, exclusively with the inflaton. It is justified by the realization of the virial equlibrium for this field.

In Fig. 3 the EoS of all three degrees of freedom are shown. It is clear, that they possess an EoS from rather early times, promptly after the large amplitude $r(t, \mathbf{x})$ oscillations are damped.They are nearly linear, of the form $p=w \rho$. The cooling pushes the system through the points of the EoS at the pace of the expansion, therefore the different points can be labeled by the instant when the system passes through them.

The inflaton and the Higgs field have nearly the same mass of the order of the GUT scale. Therefore we expect them (after the virial "equilibrium" is reached) to follow nearly the same $p-\rho$ smooth line, with the decrease of the energy density due to the expansion. Since the inflaton is almost decoupled one expects for it $w \approx 0$, and finds at early times $w \sim 1 / 10$. The Higgs field starts with a slightly larger slope. We note, that the linear regime is reached the slowest by the Higgs field, and also its trajectory in the $p-\rho$ plane displays noticeable quantitative lattice spacing dependence.

The Goldstone oscillations obey after virialization a perfect radiative $\operatorname{EoS}\left(p_{G}=w_{G} \rho_{G}, w_{G} \approx 1 / 3\right)$. For larger energy densities $\left(\rho_{G} \geq 18-23\right)$, that is for earlier times $(85 \leq t \leq 140)$, one observes a slight deviation from the slope $1 / 3$. As one sees in the right hand plot of Fig 3 the curve of $w_{G}$ determined with help of the space averages over the Goldstone densities (9) approaches $w_{\text {rad }}=1 / 3$ rather smoothly. Practically no dependence on the lattice spacing can be observed.

This smooth functional form allows a simple quantitative estimate for the composition of the 

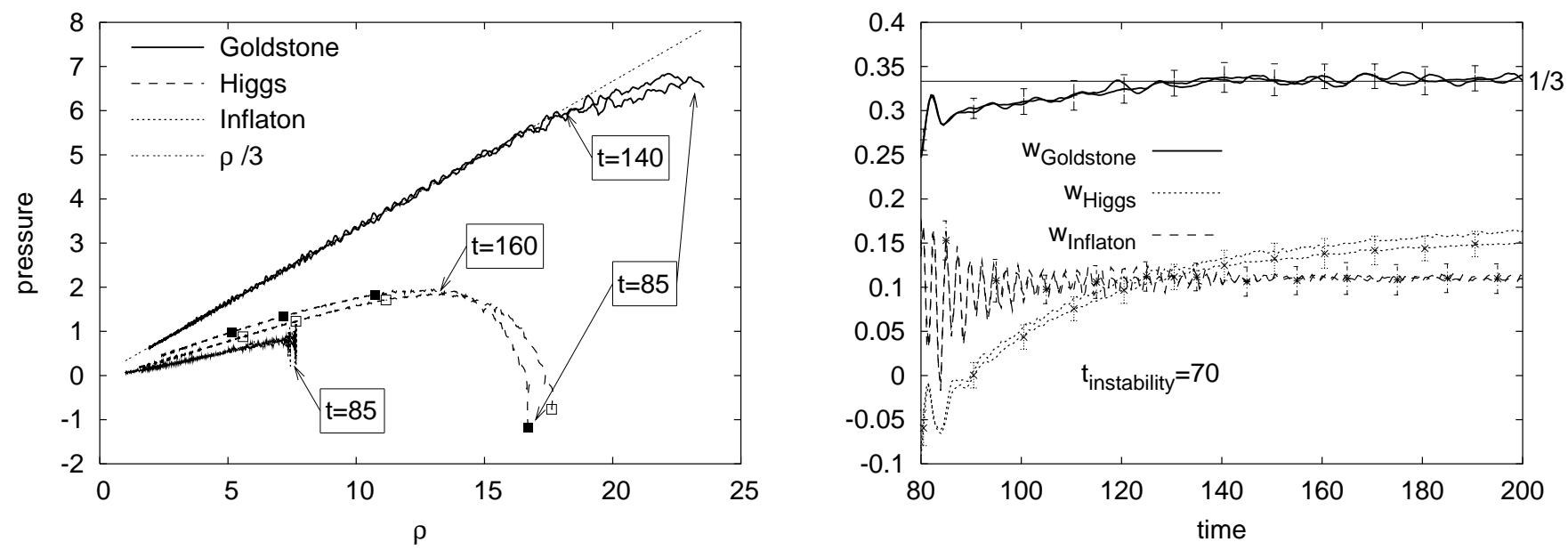

Figure 3: Equations of state (EoS) for the independent degrees of freedom $\left(g=0.1, \lambda=3 g^{2}\right)$. Left, the $p(\rho)$ trajectories are shown for the three independent degrees of freedom. The moments of time, where the linear regime, characterized by the constant $w=p / \rho$ sets in are indicated next to the curves. The twin curves display results obtained on lattices of equal physical size, but of different lattice spacing $\left(N=64, \delta x_{l a t}=1\right.$ (open square), $N=128, \delta x_{l a t}=0.5$ (full square)). The curves represent the average of 20 runs for $N=64$ and 10 for $N=128$. In the right figure the evolution of the $w_{i}=p_{i} / \rho_{i}$ ratio for the different physical degrees of freedom is shown, $(i=G, H$, inflaton $)$.

"Goldstone gas", if one assumes that it consists of a noninteracting mixture of elementary gapless Goldstone waves and of nonrelativistic heavy objects composed of coherent configurations of the angular degree of freedom of the $O(2)$-field. The measured ratio $\rho / p$ is given then by $3(1+y), y=$ $\rho_{\text {heavy }} / \rho_{\text {elementary }}$. This ratio smoothly approaches zero around $t|m| \sim 140$, till when the decay of the heavy extended objects will be complete on both the $N=64$ and the $N=128$ lattices. The time dependence of the ratio $\rho_{\text {heavy }} / \rho_{\text {full Goldstone }}$ is compatible with an exponential decay with a lattice spacing independent rate: $22(4)|m|^{-1}$. This fit was restricted to the range $100<t|m|<200$, where the EoS is already well defined and the energy density of the heavy objects is above the noise level. We expect that these topological objects were created during the tachyonic instability, hence at the time the EoS is stabilized some of them might have decayed already. As an estimate for the initial energy density confined to topological objects we extrapolated the exponential time dependence back to the moment of the instability $(t \approx 70)$ and found that (for both lattice spacings realizing the lattice size: $\left.N \delta x_{l a t}=64\right)$ slightly more than one third of the energy density of the angular motion was concentrated in heavy objects and two thirds were carried by massless quasiparticles. A polynomial fit to $w_{G}(t)$ would give an even lower estimate for $y(t=70)$. This analysis substantiates our claim that direct Goldstone production dominates in the tachyonic preheating.

One finds complementary information on the Goldstone evolution from Fig. 1. The gradient energy density grows higher than the kinetic energy as the Goldstone field gets excited. This excess reflects the formation of topologically characterizable extended objects. In Fig. 1 we also showed the average of the integral of $\delta \varphi(x)$, which is the variation of the angular orientation of the complex field from one site to the next one along straight lines parallel to the three axes. This integral is very large before the instability. After a sudden drop suffered at the moment of the tachyonic instability, it continues to decay gradually. This evolution goes parallel with the disappearance of the gradient 


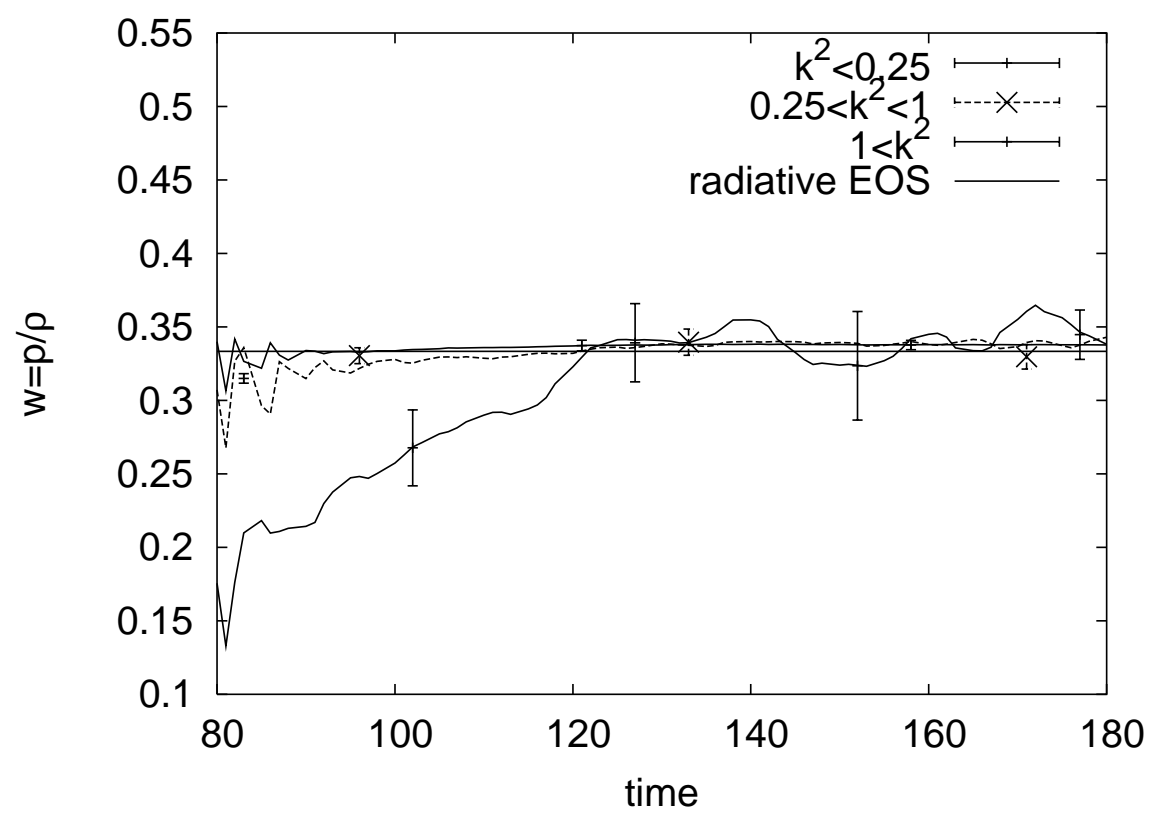

Figure 4: The evolution of the EoS of the Goldstone oscillators in three different Fourier regions. The curves show the average over eight runs for $N=64$, the calculated error of the average is signalled by the error bars.

energy excess.

In order to deepen the understanding of the role played by these topological objects we disentangle their contribution to the energy density and the energy fraction carried by the "elementary" Goldstone quasiparticles. The topological objects contribute only in the low $k$ region of the Fourier space. In order to realize this idea, the $k$ space of the Goldstone degrees of freedom was splitted into three characteriztic regions: $k^{2} /|m|^{2}=\left[(0,0.25),(0.25,1.0),\left(1.0, k_{\text {cutoff }}^{2}\right)\right]$. Separate EoS were fitted in the three regions. In Fig. 四 one sees that the deviation from the radiative EoS is localized to the lowest $k$ region. (The curve is rather insensitive to the exact choice of the values of the separating wave numbers). From a linear fit to $w_{G \text {,lowk }}(t)$ on the interval $80 \leq|m| t \leq 120$, one extrapolates for $t=70 \rho_{\text {heavy }}\left(k^{2} \leq 0.25|m|^{2}\right) / \rho_{G}\left(k^{2} \leq 0.25|m|^{2}\right) \approx 0.5$.

\section{On the mechanism of tachyonic mode excitation}

In hybrid inflationary scenarios the possible mechanisms for the Higgs field excitation were already discussed at length in the literature [15, 16, 19]. In this paper we concentrate on the angular component of the matter field and on the inflaton.

The excitation of the Goldstone and the inflaton field is driven by the Higgs field. It turns out that the gradient energy density of $r(\mathbf{x}, t)$ is about five times smaller than its kinetic energy density during the instability interval, therefore it is reasonable to replace $r^{2}(\mathbf{x}, t)$ in the field equations of the Goldstone and of the inflaton fields by its spatial average, ${\overline{r^{2}}}^{V}$.

The analysis is particularly simple for the inflaton, because in this approximation the equations of its spatial Fourier components are linear. According to the proposed model the symmetry breaking 
simply increases the effective squared masses of these uncoupled oscillators

$$
\ddot{\sigma}_{\mathbf{k}}+\omega_{\mathbf{k}}^{2}(t) \sigma_{\mathbf{k}}=0, \quad \omega_{\mathbf{k}}^{2}(t)=\mathbf{k}^{2}+m_{\sigma}^{2}+g^{2} \bar{r}^{V} V(t) .
$$

Since we are interested in the excitation mechanism, which is rather unaffected by the overall expansion $\left(H^{-1} \gg \Delta t_{\text {reheating }}\right)$ we dropped the derivatives of the scale factor in these equations.

One can construct the energy balance of each mode by integrating the equation

$$
\frac{d E_{\mathbf{k}}}{d t}=\frac{1}{2} \frac{d \omega_{\mathbf{k}}^{2}}{d t} \sigma_{\mathbf{k}}^{2}
$$

which yields

$$
E_{\mathbf{k}}(t)-E_{\mathbf{k}}(0)=\frac{g^{2}}{2} \int_{0}^{t} d t^{\prime} \frac{d \bar{r}^{2} V}{\left.d t^{\prime}\right)} \sigma_{\mathbf{k}}^{2}\left(t^{\prime}\right) .
$$

If one sums up the equations for all $\mathbf{k}$, one finds

$$
E_{\text {inflaton }}(t)-E_{\text {inflaton }}(0)=\frac{g^{2}}{2} \int_{0}^{t} d t^{\prime} \frac{d \bar{r}^{2}\left(t^{\prime}\right)}{d t^{\prime}}{\overline{\sigma^{2}}}^{V}\left(t^{\prime}\right)
$$

This result may be easily compared to the full numerical solution. The agreement is quite spectacular when one fills the Brillouin-zone completely, and deteriorates when $k_{\max }$ is decreased. The approximation based on homogenous Higgs fluctuations is less appropriate for the lower- $k$ inflaton modes.

A similar approximate construction might be attempted for the Fourier transform of the angular variable, which leads to the equation:

$$
\ddot{\varphi}_{\mathbf{k}}(t)+2 \frac{d}{d t} \overline{\ln r(\mathbf{x}, t)}^{V} \dot{\varphi}_{\mathbf{k}}+k^{2} \varphi_{\mathbf{k}}=0 .
$$

This is a set of equations for independent oscillators damped by a common friction. An equation Eq. (15) could in principle account for the delay in the excitation of the Goldstone kinetic energy (see Fig. (1) since the short but strong common friction effect nearly stops the initially independent angular oscillation modes $\left(\dot{\varphi}_{\mathbf{k}} \approx 0\right)$ in the same instant. When $\overline{\ln r}^{V}$ is stabilized, the friction disappears and all oscillators start to move with equal phase angle.

Both the friction coefficient $\frac{d}{d t} \overline{\ln r(\mathbf{x}, t)}^{V}$ and the initial conditions for $\varphi_{\mathbf{k}}$ were taken from the numerical solution of the full dynamics. The energy density resulting from the solution of Eq. (15) produced much less excitation in the Goldstone modes than one observes in the full solution of Eq. (11). This forces us to conclude that the inhomogeneity of $r(\mathbf{x}, t)$ plays important role also in the Goldstone excitation. This conclusion seems to depend rather sensitively on the value of the couplings $\lambda, g^{2}$. The smaller is $\lambda$ the more important is the inhomogenous contribution to the Higgs kinetic spectra already in the first oscillation period after the spinodal instability.

\section{Expansion and late-time cooling}

The variation of the cosmological scale factor directly after the instability reflects radiation domination. We find by a high quality power law fit to its numerical evolution the behavior

$$
a(t) \sim(t-\text { const. })^{\gamma}, \quad \gamma=0.54(2),
$$


where the error is estimated from the standard deviation of the fitted $\gamma$ values when the time interval $t|m|=100-1000$ is splitted into several shorter intervals. One may note that the value of $\gamma$ in the time interval (100-2000) is reasonably independent of the lattice constant. The variation of the scale factor is $a(|m| t=2000) / a_{\text {init }} \approx 2.5$. The lattice artefacts appear for $|m| t>2000$. This exponent, however, shows a time dependence on longer time scales as it approaches $\gamma_{\text {matter }}=2 / 3$. This experience shows that special care must be taken if one wishes to enforce a simple power law behavior for $a(t)$ over an extended time interval as it was done in Refs. [5, 6, 19].

If the equations of state are linear, then the actual rate of expansion conforms to the equation of state of the mixture system:

$$
\begin{gathered}
p_{\text {full }}(t)=w_{\text {full }}(t) \rho_{\text {full }}(t), \quad \gamma(t)=\frac{2}{3\left(1+w_{\text {full }}(t)\right)}, \\
p_{\text {full }}=p_{\text {Goldstone }}+p_{\text {Higgs }}+p_{\text {inflaton }}, \quad \rho_{\text {full }}=\rho_{\text {Goldstone }}+\rho_{\text {Higgs }}+\rho_{\text {inflaton }} .
\end{gathered}
$$

This $w_{\text {full }}$ coefficient is a subject of continuous shift from $w_{\text {full }} \approx 0.23(1)$ towards zero, which would mean the ideal matter dominated EoS. For the time interval of the fit Eq. (16) the time-averaged value $\overline{w_{\text {full }}}=0.22(2)$ agrees well with the fitted $\gamma$ exponent.

For completely decoupled field components one would expect

$$
3\left(1+w_{i}(t)\right)=-\frac{d \ln \rho_{i}(t)}{d \ln a(t)}, \quad i=\text { Goldstone, Higgs, inflaton }
$$

with an (almost) time independent $w_{i}$ value. They should agree with the corresponding coefficients of the different EoS, if the temporal evolution consists simply of the expansion of the noninteracting gas components. Indeed, this is the case of the inflaton, but the $O(2)$ sector shows rather large deviations. From Eq.(18) one finds that the Goldstone energy density on the average decreases more slowly with $a(t)$ than expected for a massless radiation. A similar comparison reveals that the rate of cooling of the Higgs mode is faster than for a nonrelativistic gas. The powers $\gamma_{i}(t)$ vary with time quite strongly. These tendencies indicate an energy transfer to the angular motion in the $O(2)$ configurational space from the radial one. The situation is puzzling, since the average energy density of the Goldstone modes is higher than that of the radial oscillations.

The investigation of the kinetic power spectra provides much more detailed information. One finds that the Higgs and inflaton degrees of freedom quickly approach classical equipartition as suggested by their EoS. The power spectra of the Goldstone degree of freedom reveal interesting regularities. Fig. 5 shows the Goldstone energy spectra multiplied by $a^{4}(t)$ at late times (well within the regime of virial equilibrium). The shape near the origin is very similar to what was found in Minkowski metric [12. The durable deviation from equipartition appears as a result of anomalously slow relaxation in the lowest comoving $k$ region, frozen in an almost instant decoupling after the tachyonic instability. This is demonstrated by the fact that this part of the spectra shows perfect $a^{-4}(t)$ scaling. The height and the width of this decoupled out-of equilibrium spectra in the low- $k$ region is invariant under a factor-of-two change of the lattice spacing, therefore the early decoupling is certainly a physical effect.

The Goldstone energy density in the high- $k$ region is much lower but it gradually increases and approaches some sort of equipartition. This rise is apparently fed by the uniform decrease of the power of the radial (Higgs) motion. The $k_{\lim }$ value separating the region where the spectral power of the Goldstone is already stationary from the part which is still increasing is shifted gradually towards 


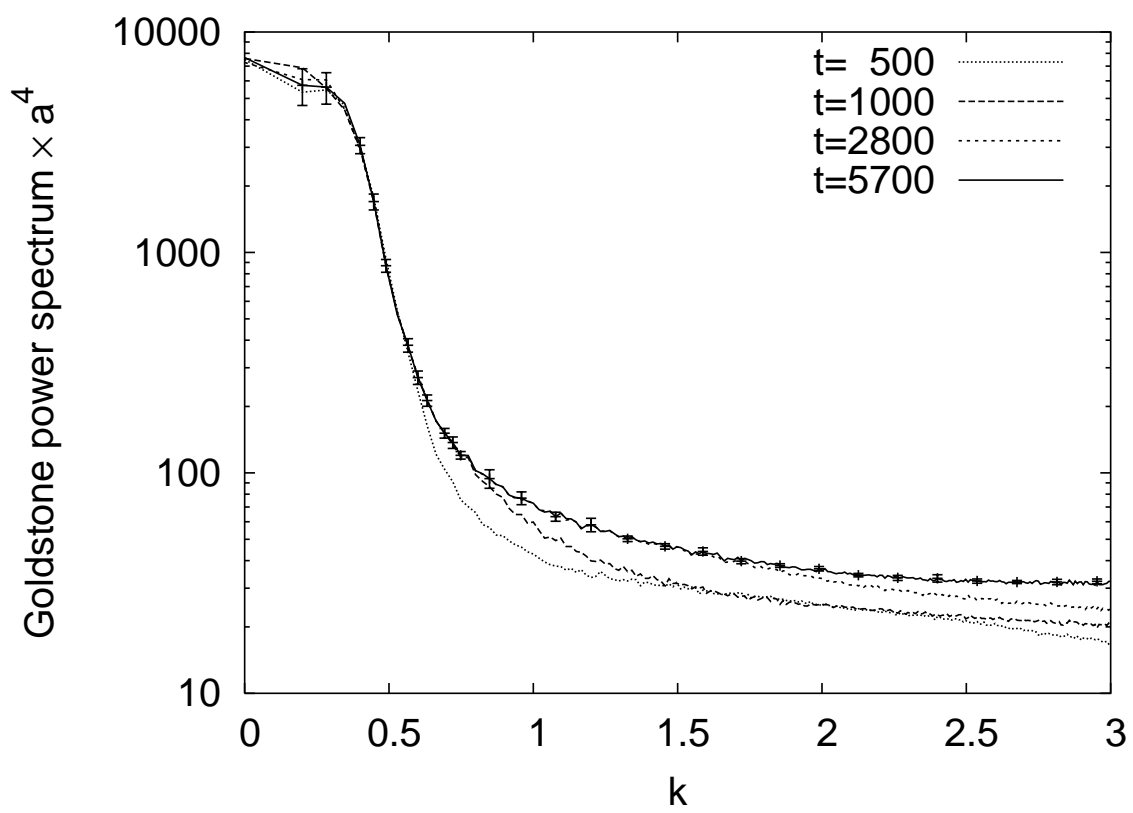

Figure 5: Time evolution of the kinetic energy spectra of the Goldstone excitations displayed as a function of the comoving wave number. Average over 8 runs with typical standard deviations as error bars for one of the spectra are shown. The rescaled late time spectra agree within the standard deviation in an interval $\left(0, k_{\text {lim }}\right)$ which increases with time $(g=0.1, N=64)$.

the cutoff. The final uniform level of excitation in the high- $k$ region is lower for finer lattices of the same physical size. This is easy to interpret by noticing that the same initial energy density is now ditributed when equipartition is approached among different numbers of degrees of freedom.

The synchronized variation in the Higgs and Goldstone power spectra described above has a simple physical explanation. The massive Higgs waves can decay into energetic pairs of elementary Goldstone modes irreversibly, even if their temperature is lower. In the language of conformally transformed variables the radial mass term scales with $a(t)$. We find $k_{\text {lim }}(t) a_{\text {init }} / a(t)$ to be approximately constant, and its value corresponds to the equality: $2 k_{\lim }(t) a_{\text {init }} / a(t) \approx m_{H}$. The main distinction between the two regions of Goldstone modes consists in the circumstance, that the low-(comoving)-k modes do not receive energy input from Higgs-to-Goldstone pair creation. As $k_{\text {lim }}$ sweeps through the Brillouinzone completely decoupled Goldstone modes are left behind. The physics of the system becomes seriously distorted at times when $m_{H} a(t) / a_{\text {init }}$ approaches $2 k_{\text {cutoff }}$, beyond which the Higgs waves will be artificially stabilized. This lattice spacing sensitivity is manifested in the level of excitation only for $|\mathbf{k}|>1$.

\section{Conclusions}

In this study we focused on the very early stage of the field evolution following the tachyonic instability occuring in simple realizations of the hybrid inflationary scenario. The results were shown overwhelmingly insensitive to the choice of the lattice spacing if the inhomogenous modes were filled initially only up to a maximal wave number. Its value was chosen so, that the energy density of the 
system was kept the same for different lattice spacings. Lattice spacing independence of the main observables was achieved without imposing any further normalization condition.

We found that the direct production of Goldstone excitations is very efficient. By a thorough analysis of the low- $k$ part of the Goldstone spectra and its contribution to the equation of state one can separate the elementary waves from the extended objects (strings) formed from coherent Goldstone configurations. The decay of the strings can be followed through the temporal variation of the corresponding $w_{G}=p_{G} / \rho_{G}$ ratio. The smaller is the selfcoupling $\lambda$ the longer the string-network lives.

The simple-minded model of the motion of Goldstone and inflaton modes, consisting of independent oscillators moving in the background of homogenous time-dependent Higgs fluctuations provides a semiquantitative interpretation of the excitation of these degrees of freeedom.

Curiously, we also found an interesting mechanism for energetic reheating in our investigation which still avoids the restoration of the symmetry. It might happen that some kind of strongly out of equilibrium dynamics (in our case the tachyonic instability) excites a certain degree of freedom much more efficiently (in our case the Goldstone's) than the order parameter. If their interaction is weak relative to the Hubble expansion rate, decoupling occurs very early and the matter field cannot climb out from the symmetry breaking minimum. The Fourier power spectra of the Goldstone mode is frozen in a strongly out of equilibrium shape, displaying the enhancement of low- $k$ modes. One has to question if the decoupling is sensitive to the choice of the lattice spacing. We have checked that the efficient Goldstone excitation is followed by quick decoupling preserving the same shape for the power spectra in the $|\mathbf{k}|<1$ region for $\delta x_{\text {lat }}=0.25,0.5,0.75,1.0$.

During the later transition period from radiation towards matter domination the decay of the Higgs waves into Goldstone modes can be observed. The spectra did show evidence for a lattice spacing dependence in the $|\mathbf{k}|>1$ region.

The features of the late time dynamics in the $\mathrm{O}(2)$ sector, analyzed above can be summarized in a spectral variant of the system of coupled equations, which describes the variation of the energy densities contained in (radiation like) light modes coupled to a heavy degree of freedom [14]. The variation in conformal time of the comoving Goldstone mode energies is affected by the decay of the Higgs waves into Goldstone particles. By the assumption that the momentum distribution of the Higgs degree of freedom obeys at each instant the equipartition rule, one conjectures the following kinetic equations:

$$
\begin{aligned}
\frac{d}{d \eta}\left(\rho_{G}(\mathbf{k}) a^{4}(t)\right) & =\Theta\left(|\mathbf{k}|-\frac{1}{2} m_{H} a(t)\right) \tau^{-1} \frac{3}{4 \pi} \frac{1}{k_{\text {cutoff }}^{3}} \rho_{H} a^{4}(t), \\
\frac{d}{d \eta}\left(\rho_{H} a^{3}(t)\right) & =-\left(1-\frac{1}{8} \frac{\left(m_{H} a(t)\right)^{3}}{k_{\text {cutoff }}^{3}}\right) \tau^{-1} \rho_{H} a^{4}(t)
\end{aligned}
$$

( $\tau^{-1}$ is the decay rate of the Higgs waves).

The next stage of our project is to extend the investigation to the case of gauged models of hybrid inflation 19, 20, 21, which differs in very important aspects from the models where Goldstone modes appear. 


\section{Acknowledgements}

The authors enjoyed very informative discussions with A. Jakovác and Zs. Szép. Remarks by M. Hindmarsh at SEWM'02 are gratefully acknowledged. This research was supported by the Hungarian Research Fund (No. T037689).

\section{References}

[1] A.D. Linde, Phys. Rev. D49 (1994) 748

[2] J. Garcia-Bellido and A.D. Linde, Phys. Rev. D57 (1998) 60557

[3] R.L. Davis, Phys. Rev. D32 (1985) 3172

[4] D.N. Spergel, N. Turok, W.H. Press and B.S. Ryden, Phys. Rev. D43 (1991) 1038

[5] M. Yamaguchi, Phys. Rev. D60 (1999) 103511

[6] M. Yamaguchi, J. Yokoyama and M. Kawasaki, Phys. Rev. D61 (2000) 061301(R)

[7] G. Felder, J. Garcia-Bellido, P.B. Greene, L. Kofman, A.D. Linde and I. Tkachev, Phys. Rev. Lett. 87 (2001) 011601

[8] G. Felder, L. Kofman and A.D. Linde, Phys. Rev. D64 (2001) 123517

[9] D. Boyanovsky, H.J. de Vega, R. Holman, D.S. Lee, and A. Singh, Phys. Rev D51 (1995) 4419

[10] D. Boyanovsky, R. Holman and J.F.J. Salgado, Phys. Rev. D54 (1996) 7570

[11] D. Boyanovsky, D. Cormier, H.J. de Vega and S. Prem Kumar, Phys. Rev. D57 (1998) 2166

[12] Sz. Borsányi, A. Patkós and D. Sexty, Phys. Rev. D66 (2002) 025014

[13] M. Yamaguchi and J. Yokoyama, hep-ph/0210343

[14] E.W. Kolb and M. Turner, The Early Universe, Addison-Wesley 1990, New York

[15] T. Asaka, W. Buchmüller and L. Covi, Phys. Lett. B510 (2001) 271

[16] J. Garcia-Bellido, M. Garcia Perez and A. Gonzalez-Arroyo, hep-ph/0208228

[17] Sz. Borsányi, A. Patkós and D. Sexty, hep-ph/0301117, to appear in Procs. of SEWM'02, Sept. 29-Oct. 2 2002, Heidelberg, ed. M.G. Schmidt

[18] M. Sallé, J. Smit and J.C. Vink, hep-ph/0112057

[19] E.J. Copeland, S. Pascoli and A. Rajantie, Phys. Rev. D65 (2002) 103517

[20] J. Skullerud, J. Smit and A. Tranberg, hep-ph/0210349, to appear in Procs of SEWM'02, Sept 29-Oct. 2 2002, Heidelberg, ed. M.G. Schmidt

[21] J. Garcia-Bellido, M. Garcia Perez and A. Gonzalez-Arroyo, hep-ph/0304285 